\title{
Ectopic Expression of Ephrin-A5 Under the EphA8 Promoter at the Anterior Region of the Superior Colliculus
}

\author{
Hyuna Noh and Soochul Park* \\ Department of Biological Science, Sookmyung Women's \\ University, Seoul 140-742, Korea
}

\begin{abstract}
EphA/ephrin-A mediated signaling has emerged as a key mechanism regulating axon guidance and topographic mapping, particularly in the well-characterized visual system from the retina to the superior colliculus (SC). In this study, EphA8 bacterial artificial chromosome (BAC) was manipulated to contain a floxed eGFP and human ephrin-A5 expression cassette using homologous recombination method. In the mice containing the recombinant BAC, it was shown that GFP is expressed in an anterior > posterior gradient in the SC. Furthermore, when these mice were crossed with the transgenic mice expressing Cre under the EphA8 promoter, it was evident that a GFP expression cassette was eliminated, and that human ephrin-A5 was ectopically expressed in the anterior region of the SC. This transgenic model would be useful to analyze the role of ephrin-A5 in the SC during the retinocollicular topography formation.
\end{abstract}

Key words: ephrin-A5, EphA8, superior colliculus, topographic mapping

\section{INTRODUCTION}

Recent studies indicate that Eph receptors and ephrins coordinate various developmental processes but also physiological events and homeostasis of diverse adult organs (Klein, 2004; Pasquale, 2008). These emerging Eph-ephrin functions suggest that the unbalanced signaling of these molecules may lead to the genesis of a variety of diseases such as diabetes and cancer. An intriguing feature of Eph/ ephrin signaling is that Ephs and ephrins are mutually exclusively expressed and that they elicit bidirectional signaling between two opposing cells

*To whom correspondence should be addressed.

TEL: 82-2-710-9330, FAX: 82-2-715-9331

e-mail: scpark@sookmyung.ac.kr

Received June 10, 2010

Accepted for publication June 22, 2010 through cell-cell contact (Davy and Soriano, 2005). However, in some cases, Ephs and ephrins are co-expressed in the same cells. For example, it was shown that co-expression of ephrin-As with EphAs in the nasal retinal ganglion cells reduced the sensitivity of EphAs towards ephrinAs expressed in the posterior region of the superior colliculus (Hornberger et al., 1999). In addition, formation of the cis Eph-ephrin complex within the same cell was shown to cause an abolishment of the tyrosine phosphorylation of EphA3, thereby leading to a loss of sensitivity towards ephrin-As applied in trans (Carvalho et al., 2006). These results suggest that co-expression of Ephs and ephrins impairs their signaling through cis-interaction. In contrast, another study demonstrated that EphAs and ephrinAs are localized to different membrane domains and signal independently. They 
showed that EphA forward signaling directed growth cone collapse and retraction while ephrinA reverse signaling motor axon growth and attraction (Marquardt et al., 2005).

In this study, we generated a transgenic mice ectopically expressing ephrin-A5 at the anterior superior colliculus (SC) where various EphA receptors are normally expressed with anterior $>$ posterior gradient (Lemke and Reber, 2005). These mice may be a valuable tool to elucidate whether ephrin-As acts as an inhibitor or independent ligand in the anterior SC during the retinocollicular topography formation.

\section{MATERIALS AND METHODS}

\section{Generation of BAC transgenic mice}

Construction of targeting vector, homologous recombination and Southern analyses were performed as described previously (Kim et al., 2007). BAC transgenic mice containing EphA8-eGFP were genotyped by PCR using the following primers: 5'-TCTTGCCAGCTCTACCACCA-3' and 5'-CAGAGAACTTCAGCGGTCCA-3'. BAC transgenic mice containing EphA8-Cre mice were also identified by PCR using the primers 5'-CAGGATATCGTAATCTGGCATTTC-3' and 5'-ATGACAATATTTACATTGGTCCAGC-3'.

\section{Histology and immunohistochemistry}

The mice were perfused with 4\% PFA in $1 \times$ PBS and the brains were post-fixed for $2 \mathrm{hr}$ in cold $4 \%$ PFA in 1× PBS, washed three times for $20 \mathrm{~min}$ each with PBS, incubated in $30 \%$ sucrose in $1 \times$ PBS for overnight at $4^{\circ} \mathrm{C}$ and then embedded in tissue freezing medium (Jung). Sections $(20 \mu \mathrm{m})$ were stored at $-20^{\circ} \mathrm{C}$ or $-80^{\circ} \mathrm{C}$. For the antibody staining, the sections were washed two times in $0.1 \%$ Triton X-100/PBS (10 minutes each), blocked with $10 \%$ Horse serum, $0.1 \%$ Triton X-100/PBS $1 \mathrm{hr}$ at room temperature. The primary antibody was diluted in blocking solution and incubated at $4^{\circ} \mathrm{C}$ overnight. Goat anti-human ephrin-A5 antibody was purchased from $R$ and $D$ system. After three times washes in $0.1 \%$ Triton X-100/PBS (30 minutes each), the secondary antibody, diluted in blocking solution, was applied for $3 \mathrm{hr}$ (FITC-conjugated rabbit anti-goat, chemicon, $1: 200)$. After three washes with $0.1 \%$ Triton X-100/PBS, counterstaining was performed with DAPI and the sections were embedded with vectasheild (Vectorlab).

\section{RESULTS}

Construction of a recombinant EphA8 bacterial artificial chromosome (BAC) containing a eGFP-ephrinA5 expression cassette

Previous studies showed that co-expressed Eph receptors and ephrin ligands negatively regulate its biological function or mediate opposing actions on their counter-partners presented in trans (McLaughlin and O'Leary, 2005). To investigate whether coexpression of ephrin-A5 in the EphA8-expressing superior collicular cells affects the retinocollicular topography, we modified a EphA8 BAC clone, RP23-357K18, which is shown to express LacZ reporter gene in the anterior region of the superior colliculus (SC) but not in the retina (Kim et al., 2007). Because the EphA8 gene contains 17 exons, is about $28 \mathrm{~kb}$ in length and located in the center of the EphA8 BAC clone, it is expected that RP23-357K18 clone contains the regulatory elements and locus control regions necessary to recapitulate the endogenous expression of EphA8 in vivo.

In order to insert the eGFP-ephrinA5 expression cassette into the first exon of EphA8 in the BAC clone, we constructed a targeting vector containing a $1.2 \mathrm{~kb}$ EphA8 genomic DNA upstream of the first exon, eGFP flanked by two loxP sites, a human ephrin-A5 cDNA, kanamycin resistance gene flanked by two FRT sites, and a $0.7 \mathrm{~kb}$ EphA8 genomic DNA downstream of the first exon (Fig. 1). This targeting vector was transformed into the bacteria carrying the EphA8 BAC DNA and pKOBEGA plasmid containing an inducible lamda phage recombinase system. To induce the recombinases, bacteria were incubated in arabinose-containing medium, and the first recombinant BAC clones were selected in the presence of chloramphenicol and kanamycin. Southern blot analysis using GFP as a probe revealed that a $9.6 \mathrm{~kb} \mathrm{Kpnl}$ fragment was detected in the wild type BAC, whereas a 8.2 $\mathrm{kb}$ fragment was detected in the first recombinant BAC DNA (data not shown). When Southern blot analysis was performed using ephrinA5 cDNA as a probe, a $7.1 \mathrm{~kb}$ fragment was detected in the first 

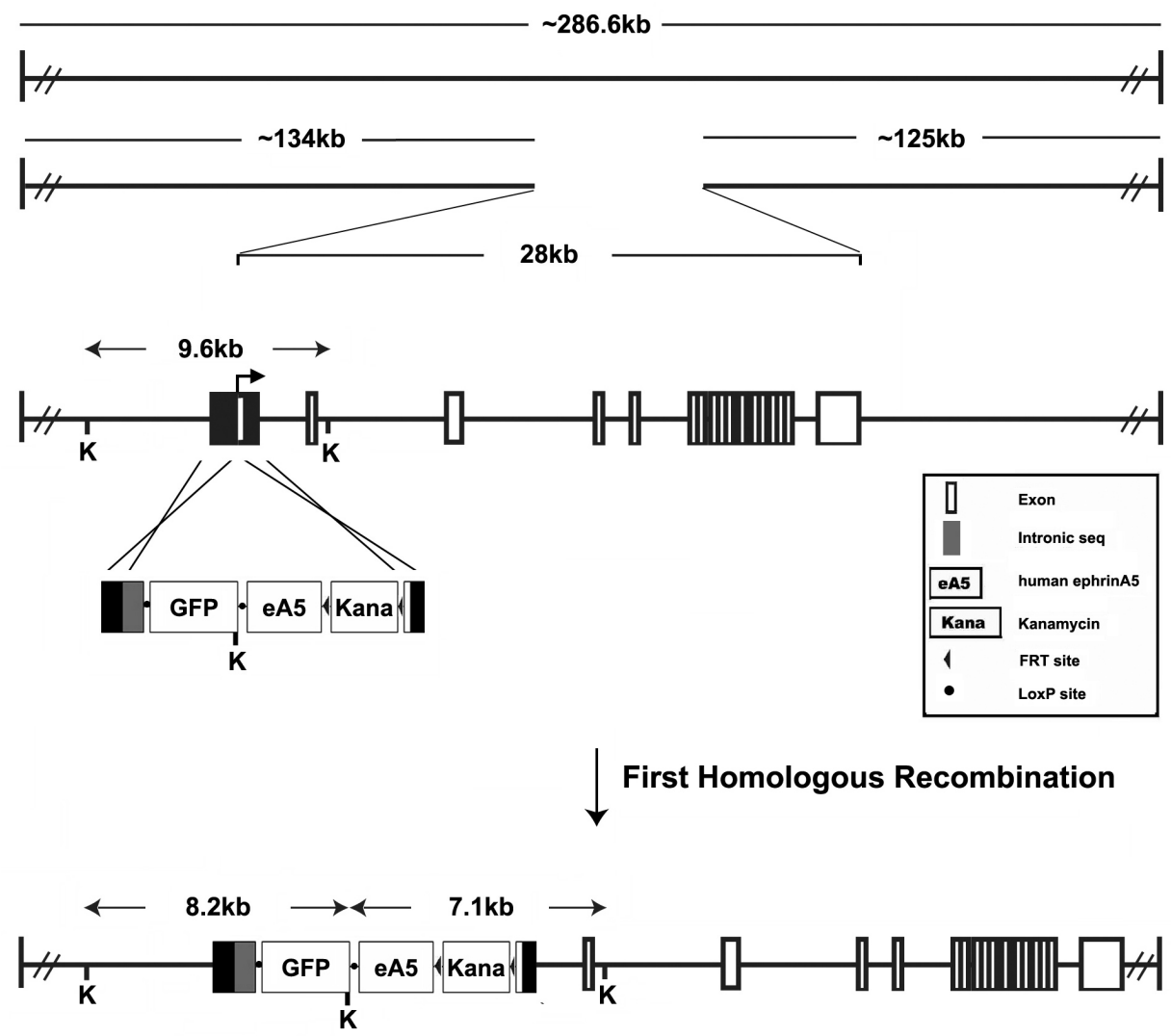

First Recombinant
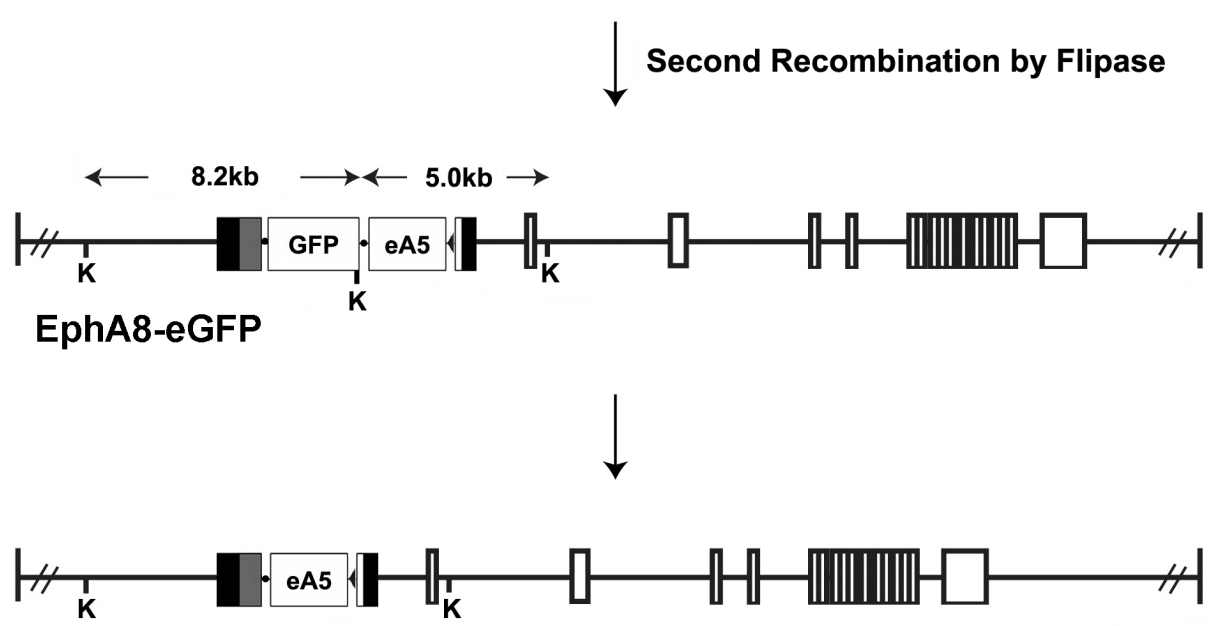

\section{EphA8-ephrinA5}

Fig. 1. Strategy for constructing a recombinant EphA8 BAC containing a eGFP-human ephrinA5 expression cassette. The EphA8 BAC clone, RP23-357K18, contains approximately $286.6 \mathrm{~kb}$ genomic DNA, and the ephA8 gene is positioned approximately in the middle of the BAC clone. The ephA8 gene is approximately 28 $\mathrm{kb}$ long as represented by a thin line, and it comprises 17 exons. The targeting vector was designed to contain two ephA8 genomic DNAs on its either side, which allow a specific homologous recombination between the targeting vector and the EphA8 BAC DNA at the genomic region surrounding the first exon. The floxed eGFP-human ephrinA5 cDNA expression cassette and kanamycin resistance gene flanked by FRT sites were also inserted in between two homologous genomic arms of the targeting vector. Through the first homologous recombination, the eGFP-human ephrinA5 cDNA and kanamycin resistance cassette was inserted into the first ephA8 exon in the EphA8 BAC clone. Then, the kanamycin resistance cassette was selectively eliminated by expressing Flipase, leading to the EphA8-eFFP BAC clone containing only the floxed eGFP-human ephrinA5 cDNA expression cassette. Finally, it was expected that the expression Cre recombinase eliminate the floxed eGFP from the EphA8-eGFP BAC clone and that the EphA8-ephrinA5 BAC express human ephrin-A5 instead of eGFP under the EphA8 promoter. K: kpnl. recombinant BAC clone, whereas a $9.6 \mathrm{~kb}$ fragment was detected in the wild type BAC clone (data not shown). To eliminate the kanamycine resistance cassette from the first recombinant BAC clone, FLP plasmid was electroporated into cells containing the first recombinant $\mathrm{BAC}$ clone, selected at $30^{\circ} \mathrm{C}$ in the presence of chloramphenicol, kanamycin and tetracycline, and then flipase was induced by shifting temperature to $42^{\circ} \mathrm{C}$ to eliminate a kanamy- cin cassette between two FRT sites. To confirm EphA8-eGFP recombinant, we performed Southern blot analysis using ephrinA5 as a probe, revealing that a $5.0 \mathrm{~kb} \mathrm{Kpnl}$ digested fragment was detected in the final recombinant BAC clone (data not shown). 

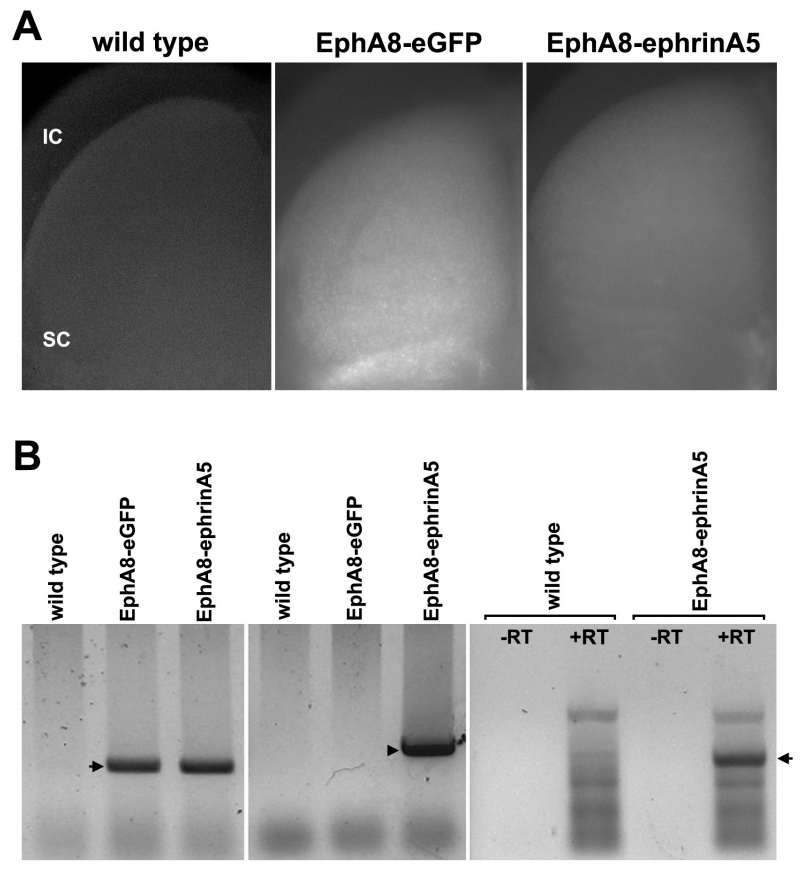

Fig. 2. Expression analysis of eGFP at the anterior region of the superior colliculus. (A) Transgenic mice containing EphA8-eGFP BAC were crossed with those containing EphA8-Cre BAC. The midbrains were isolated from each littermate at P5 and the dorsal region of each midbrain was visualized with GFP fluorescence. The wild type mice did not show any GFP signal in the SC (the first panel), whereas the EphA8-eGFP mice revealed an anterior $>$ posterior gradient expression of GFP at the SC (the second panel). In addition, GFP signal was barely detectable in the SC of the compound transgenic line containing both eGFP-ephrinA5 and Cre (the third panel). (B) PCR genotyping was performed using tail genomic DNAs derived from each littermate (the first and second panels). Primers were designed to amplify a portion of human ephrin-A5 cDNA, which is expected to produce a 450 bp PCR product in each transgenic line but not wild type mice (the first panel). Likewise, primers were designed to amplify a portion of Cre gene, which is expected to produce a $500 \mathrm{bp} \mathrm{PCR} \mathrm{product} \mathrm{only} \mathrm{in}$ a compound transgenic line containing both eGFP-ephrinA5 and Cre (the second panel). RT-PCR analysis was also performed to identify the expression of human ephrin-A5 in a compound transgenic line. RNAs were purified from the SC extract of each littermate and the same primers for human ephrin-A5 PCR genotyping (the first panel) were used for RT-PCR analysis (the third panel). The expected 450 bp RT-PCR product was detected only in the presence of RT from the SC of the compound transgenic line (lane 4). SC: superior colliculus, IC: inferior colliculus, RT: reverse transcriptase.

\section{Generation of BAC transgenic lines express- ing eGFP or human ephrinA5 under the EphA8 promoter}

Next, EphA8-eGFP BAC clone was injected into a fertilized ICR mouse zygote to generate BAC transgenic mice expressing eGFP under the EphA8 promoter. Transgenic mice containing the EphA8eGFP BAC DNA were directly confirmed by PCR

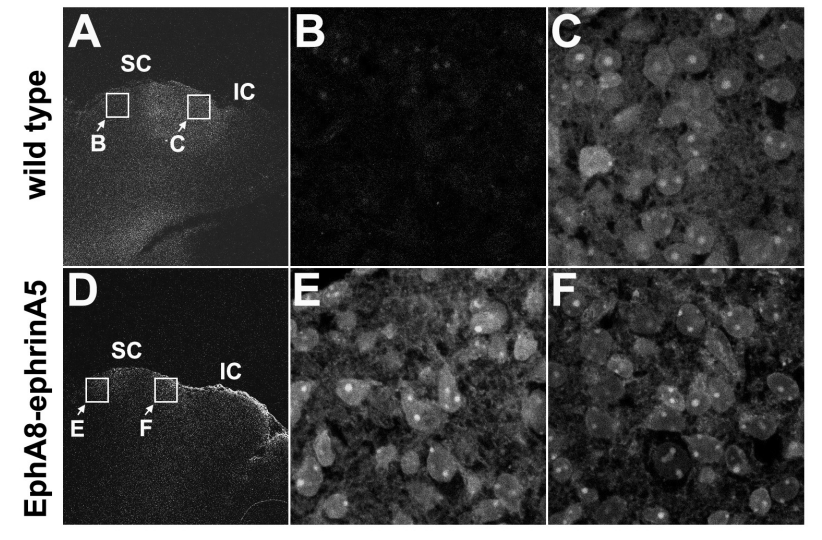

Fig. 3. Expression analysis of human ephrin-A5 at the anterior region of the superior colliculus. $(A \sim C)$ Immunohistochemical analyses of ephrin-A5 proteins in the sagittal sections of the SC from the wild type littermate. Note that the endogenous ephrinA5 was detectable in the posterior SC but not in the anterior SC. (D $\sim F)$ Immunohistochemical analyses of ephrin-A5 proteins in the sagittal sections of the SC from the transgenic littermate expressing human ephrin-A5 under the EphA8 promoter. In contrast to the wild type littermate, ephrin-A5 proteins were detectable in both anterior and posterior regions of the $\mathrm{SC}$ in the transgenic line.

genotyping as shown in Fig. 2B (first panel, lane 2). To investigate whether the transgenic line express eGFP under the EphA8 promoter, we dissected out the superior colliculus (SC) from postnatal (P) 5 day mice and then observed the GFP expression under the fluorescent microscope. As shown in Fig. 2A, eGFP was detected as an anterior > posterior gradient in the SC of the transgenic mice (the second panel), which is very similar to the endogenous expression pattern of EphA8 in the SC. In contrast, eGFP was not detectable in the SC of the wild type littermate (Fig. 2A, the first panel).

To further analyze whether the floxed eGFP in the EphA8-eGFP transgenic mice is effectively eliminated by Cre recombinase, we generated a EphA8-Cre transgenic line using the same EphA8 $\mathrm{BAC}$ clone described in Fig. 1, where P1 bacteriophage Cre DNA was specifically inserted into the first exon of EphA8. This EphA8-Cre transgenic line was crossed with the EphA8-eGFP BAC transgenic line to generate a compound transgenic line expressing both human ephrin-A5 and Cre under the EphA8 promoter (see EphA8-ephrinA5 in Fig. 1). It was evident that this compound transgenic line contained both EphA8-eGFP and EphA8-Cre BAC clones, based on PCR genotyping (Fig. 2B, 
the first and second panels, lane 3). In addition, eGFP expression was barely detectable in the SC of the compound transgenic line, indicating that the floxed eGFP expression module was efficiently eliminated by Cre recombinase in the SC (Fig. 2A, third panel). To further confirm whether human ephrin-A5 is expressed under the EphA8 promoter, we performed RT-PCR analysis using total RNAs isolated from the SC extracts, where the primers were designed to specifically amplify human ephrinA5 cDNA but not mouse ephrin-A5 cDNA. As expected, a PCR product corresponding to human ephrin-A5 gene was detected only in the SC of a compound transgenic line but not its wild type littermate (Fig. 2B, third panel, lane 4).

\section{Expression analysis of human ephrin-A5 in the anterior region of the mouse superior colliculus}

To further investigate whether human ephrinA5 cDNA in the compound transgenic mice is expressed in the anterior region of the SC, we prepared a series of cryosections for immmunohistochemistry using anti-ephrinA5 antibody. As expected, in the wild type littermate, the endogenous ephrin-A5 was abundantly expressed in the posterior region of the SC but also in the inferior colliculus (IC) (Fig. 3A, C). However, ephrinA5 was not detectable in the anterior SC of the wild type mice (Fig. 3B). In contrast, ephrin-A5 was detectable in both anterior and posterior $\mathrm{SC}$ of the compound transgenic mice (Fig. 3D F). These results demonstrate that the compound transgenic mice containing both EphA8eGFP and EphA8-Cre BAC clones do ectopically express human ephrin- A5 under the EphA8 promoter in the anterior region of the SC.

\section{DISCUSSION}

In the present study, we generated BAC transgenic mice expressing human ephrin-A5 under the EphA8 promoter. It was evident that human ephrin- A5 cDNA was ectopically expressed at the anterior part of the SC. However, unlike other studies, we were not able to observe any altered size or morphology of the SC in those transgenic mice despite the co-expression of ephrin-A5 with EphA receptors, suggesting that misexpression of ephrin- A5 does not affect cell proliferation or cell survival at least in the SC (Depaepe et al., 2005). Nevertheless, these transgenic mice might be an ideal animal model to determine the function of ephrin-A5 in the retinocollicular topography. If the ectopic ephrin-A5 acted as a barrier for the growth of EphA-expressing retinal ganglion cell (RGC) axons, it would be expected that the number of RGC axons is significantly reduced in the SC. If the ectopic ephrin-A5 acted as a ligand for EphA receptors, the retinocollicular topography would be significantly altered.

\section{ACKNOWLEDGEMENT}

This work was supported by a grant (No. 20090083149) from Korea Science and Engineering Foundation (KOSEF); a grant (No. 2009K001246) from Brain Research Center of the $21^{\text {st }}$ Century Frontier Research Program funded by the Ministry of Education, Science and Technology, the republic of Korea.

\section{REFERENCES}

Carvalho RF, Beutler M, Marler KJ, Knoll B, Becker-Barroso E, Heintzmann R, Ng T and Drescher U (2006) Silencing of EphA3 through a cis interaction with ephrinA5. Nat Neurosci 9:322-330.

Davy A and Soriano P (2005) Ephrin signaling in vivo: look both ways. Dev Dyn 232:1-10.

Depaepe V, Suarez-Gonzalez N, Dufour A, Passante L, Gorski JA, Jones KR, Ledent $C$ and Vanderhaeghen $P$ (2005) Ephrin signalling controls brain size by regulating apoptosis of neural progenitors. Nature 435:1244-1250.

Hornberger MR, Dutting D, Ciossek T, Yamada T, Handwerker C, Lang S, Weth F, Huf J, Wessel R, Logan C, Tanaka $H$ and Drescher $U$ (1999) Modulation of EphA receptor function by coexpressed ephrinA ligands on retinal ganglion cell axons. Neuron 22:731-742.

Kim Y, Song E, Choi S and Park S (2007) Engineering lacZ Reporter gene into an ephA8 bacterial artificial chromosome using a highly efficient bacterial recombination system. J Biochem Mol Biol 40:656-661.

Klein $R$ (2004) Eph/ephrin signaling in morphogenesis, neural development and plasticity. Curr Opin Cell Biol 16:580-589.

Lemke $G$ and Reber M (2005) Retinotectal mapping: new insights from molecular genetics. Annu Rev Cell Dev Biol 21:551-580.

Marquardt T, Shirasaki R, Ghosh S, Andrews SE, Carter N, Hunter T and Pfaff SL (2005) Coexpressed EphA receptors and ephrin-A ligands mediate opposing actions on growth cone navigation from distinct membrane domains. Cell 121:127-139.

McLaughlin T and O'Leary DD (2005) Molecular gradients and development of retinotopic maps. Annu Rev Neurosci 28: 327-355.

Pasquale EB (2008) Eph-ephrin bidirectional signaling in physiology and disease. Cell 133:38-52. 